\title{
Comment les professionnels de santé confrontés à l'émergence de nouvelles arboviroses gèrent l'interface santé / environnement
}

\author{
N. Vernazza-Licht ${ }^{1}$, D. Bley ${ }^{1}$ et J. Raude ${ }^{2}$ \\ ${ }^{1}$ UMR 7300 ESPACE, France \\ 2 École des Hautes Études en Santé Publique, France
}

\begin{abstract}
Résumé. Cet article examine, à partir d'entretiens conduits à Nice en 2011 auprès de professionnels de santé, comment les pharmaciens d'officine interrogés perçoivent la gestion du risque de maladies transmises par Aedes albopictus. L'analyse porte notamment sur les discours produits sur la nuisance, l'usage de répulsifs, la question de l'information et de façon plus générale sur le rôle d'acteur de santé publique que peut jouer le pharmacien dans la gestion et la prévention des arboviroses.
\end{abstract}

En France si le moustique Aedes albopictus, vecteur du chikungunya et de la dengue, a été détecté dès 1999 dans un village de l'Orne (Basse Normandie) on le retrouve depuis 2004 dans le sud de la France en provenance probablement d'Italie. Initialement présent dans un secteur géographiquement limité au littoral méditerranéen (Alpes Maritimes 2004, Haute Corse 2006, Corse du Sud et Var 2007) il a étendu son territoire en 2009 à l'arrière-pays de ces départements. Les densités vectorielles observées sont jugées importantes [1]. En septembre 2010 deux cas autochtones de dengue ont été rapporté à quelques jours d'intervalle à Nice.

Le premier, le 9 septembre 2010, auprès d'un homme adulte n'ayant pas voyagé et le second, le 17 septembre, chez un jeune homme vivant à proximité de l'endroit où vivait le premier patient atteint. La transmission serait liée, pour les entomologistes, à un voyageur non identifié arrivant des Antilles où sévissait alors une épidémie de dengue. A la même période (24 et 25 septembre 2010), deux cas de chikungunya autochtones ont été rapportés à Fréjus, dans le Var, chez deux filles de 12 ans et la transmission serait en lien avec la proximité d'une de leur collègue revenue des Indes et atteinte du chikungunya.

Depuis 2006, et consécutivement à l'épidémie de l'Ile de la Réunion, un dispositif de lutte contre le risque de dissémination de la dengue et du chikungunya a été mis en place en France métropolitaine (surveillance des populations d'insectes, pulvérisation d'insecticide et de larvicide si nécessaire, informations des populations sur la conduite à adopter en matière de gites larvaires). Dans les départements d'implantation de l'Aedes albopictus il existe maintenant un signalement accéléré des cas suspects de chikungunya et de dengue sans attendre leur confirmation biologique. Chaque cas suspect est à notifier aux autorités sanitaires régionales avec l'envoi en parallèle d'un prélèvement permettant aux centres nationaux de références des arboviroses de confirmer ou non ce cas. Ceci permet à l'Entente départementale méditerranée (EID) de réaliser une prospection aux lieux de vie des cas et de mettre en place, si besoin, des actions de démoustication [1].

Le schéma régional de prévention de l'ARS PACA met en avant un souci «d'adaptation et d'amélioration annuelle» du dispositif de surveillance des autorités sanitaires régionales en la matière, et notamment le fait que «l'expérience acquise en PACA et Corse permettra une mise en place rapide et adaptée du dispositif dans d'autres régions » [1] ainsi que les voies de progrès qui restent à développer, et à renforcer vis-à-vis des professionnels de santé, des médias et du grand public.

Les deux cas autochtones de septembre 2010 avait fait alors passer les départements des Alpes Maritimes et du Var au «niveau $3 »$ du plan anti dissémination du chikungunya et de la dengue en métropole [2]. Ce niveau associe différentes mesures :

- le suivi quotidien des cas suspects dans les services d'urgence (Toulon, Fréjus, Saint-Raphaël pour le Var et Nice, Saint-Laurent-du-Var et Cagnes- sur -Mer pour les Alpes-Maritimes.

- le renforcement de la surveillance entomologique aux ports et aéroports et démoustication si nécessaire ;

- la sensibilisation des médecins sur la toxicovigilance vis-à-vis des insecticides,

- des mesures de lutte anti vectorielle, particulièrement dans les hôpitaux, mais également dans tous les foyers possibles de développement des larves d'Aedes albopictus.

C'est dans ce contexte que nous avons effectué une enquête qualitative à visée «exploratoire» (projet FAVELA, financement IMMI), ainsi qu'une enquête quantitative réalisée auprès de 1506 personnes (financement INPES) qui doit ensuite nous permettre de mettre en place un dispositif de recherche plus pérenne appliqué à l'ensemble de la métropole. L'objectif de ce premier travail dont nous présentons les résultats a été d'étudier l'attitude des professionnels de santé (médecins généralistes et pharmaciens d'officine) hors d'un contexte de crise et dans 
la perspective d'une situation épidémique d'une maladie à transmission vectorielle (dengue, chikungunya).

Le choix du lieu d'enquête, la ville de Nice en région PACA, était lié aux deux cas de dengue autochtones de septembre 2010, un an auparavant, et il nous semblait intéressant dans ce contexte d'apprécier la sensibilité des médecins et des pharmaciens à cette question, les interrogations éventuelles de leur clientèle, l'adéquation de la formation médicale aux maladies émergentes vectorielles. La littérature en la matière ayant mis en évidence l'existence d'une réflexion sur le rôle des pharmaciens dans la lutte anti vectorielle $[3,4]$ le choix a été fait dans cette présentation de privilégier les analyses réalisées à partir des entretiens avec des pharmaciens (officines et hospitalier).

Parmi les professionnels qui ont été rencontrés au cours du $4^{\text {ème }}$ trimestre 2011, huit pharmaciens-titulaires d'officines ont été interrogés. Ces entretiens ont été complétés par différents échanges notamment lors de la participation le $1^{\text {er }}$ décembre à Nice à une réunion de formation continue destinée aux pharmaciens du réseau PHaRE (réseau ville/hôpital de pharmaciens) ou avec des visiteuses médicales en salle d'attente.

Différentes questions ont été abordées au cours de ces entretiens :

- la situation d'exercice du professionnel de santé (âge, expérience, etc. .

- son vécu personnel et professionnel lié à la présence des moustiques, de cas de dengue autochtones dans la ville de Nice, de maladies épidémiques au niveau national

- les modalités de son savoir professionnel (connaissances, adéquation, actualisation des connaissances, informations institutionnelles etc...)

- les propositions éventuelles faites pour la gestion d'un risque d'épidémie.

\section{Le point de vue des pharmaciens}

L'objectif était en particulier d'apprécier comment les connaissances des pharmaciens et l'organisation du travail en pharmacie d'officine leur permettaient de s'adapter ou non à une situation épidémique. Deux thèses de pharmacie récentes mettent l'accent sur le rôle du pharmacien dans le contexte de la présence d'aedès albopictus. L'une s'appuie en particulier sur une enquête menée auprès de pharmaciens marseillais [3].

\subsection{Le discours sur le moustique, la nuisance et la maladie}

Les entretiens menés en fin d'été 2011 auprès de la population niçoise ont fait ressortir une présence forte de moustiques tigrés depuis quelques années et une gêne pour la population. On retrouve le même constat chez les pharmaciens même si certains estiment que la situation évolue au fil des ans. "Je pense qu'y a une recrudescence des moustiques par rapport à 20, 40 ans c'est évident» (entretien 8).

Un pharmacien explique qu'il a déjà vu des moustiques tigrés : «Les clients en avaient ramené y a deux ans, pas cette année, et puis moi j'habite en centre-ville mais avec un jardin et j'ai un voisin qui est fou d'arrosage ce qui fait qu'effectivement dans la journée j'ai des moustiques, c'est souvent des tigrés » (entretien 18).

Comme pour les champignons il est intéressant de voir que les clients apportent des «exemplaires» au pharmacien pour lui faire constater de visu ... «Oui je l'ai vu (un moustique tigre), mais on me l'a apporté un patient me l'a apporté mort, mais moi les moustiques je n'y connais pas grand-chose» (entretien 9).

Les pharmaciens parlent de moustiques, de petits (qui piquent aux chevilles et qui font mal), d'autres de plus gros témoignant de la diversité des espèces en présence, comme on avait pu le noter dans le discours de la population. Les pharmaciens disent que c'est une piqure qui est douloureuse (celle de l'Aedes albopictus) et parlent d'enfants fortement piqués que les clients amènent à la pharmacie. La majorité d'entre eux expliquent qu'ils sont gênés au plan personnel dans leur vie quotidienne et l'expliquent par la présence de zones de rétention d'eau dans le voisinage les obligeant à préférer dîner à l'intérieur les soirs d'été, à utiliser des répulsifs, à se protéger avec des sprays et des crèmes répulsives, à installer des répulsifs, voire comme l'un d'entre eux des moustiquaires autour de sa terrasse.

La présence des moustiques tigrés suscite donc des questionnements de la clientèle et des demandes de conseil en matière de traitements : «Dans les officines les gens arrivent, voilà j'ai été piqué, ça me gratte, qu'est-ce que je prends, ...» (entretien 1). On peut noter, que bien que les pharmaciens parlent tous de «nuisance», ils n'associent pas la présence des moustiques à la maladie. Ils considèrent qu'il en est de même pour leurs clients. Dans les entretiens quand ils ont associé le moustique à la crainte d'une maladie, c'est généralement en s'appuyant sur l'épisode de chikungunya à la Réunion.

Concernant le risque d'épidémie lié aux moustiques : «Je vous dis je ne suis pas épidémiologiste, je ne suis pas entomologiste mais je veux bien le croire, il y a tellement de gens qui circulent, avant il y avait la peste avec des rats qui venaient de n'importe où, maintenant avec les mouvements de population je peux penser qu'il y ait un risque» (entretien 9).

Certains estiment aussi le volume des moustiques en rapport à leurs ventes de répulsifs : «Beaucoup plus, ces deux dernières années, nous en avons eu beaucoup plus .../ça se répercute dans les ventes de répulsifs et ça se répercute aussi dans les problèmes d'infections liées à ces piqures $\gg$ (entretien 9).

La vente de répulsifs en pharmacie va évoluer en fonction de la présence perçue de moustiques, des quartiers plus ou moins exposés et de la clientèle. Par exemple, certains pharmaciens ont une clientèle «passagère, touristique » et donc une vente permanente et régulière tout au long de la saison, ce qui n'est pas le cas pour les autres.

\subsection{Les opinions sur l'usage d'insecticides tropicaux type 5/5 et la nocivité des produits}

\section{Le pharmacien et l'achat des produits insecticides}

De manière générale, c'est en dernier ressort les conditions tarifaires qui vont prévaloir sur le choix du produit en rayon. 
En effet le choix du pharmacien vis-à-vis des différentes marques de produits est guidé par le prix d'achat et en particulier quand il peut participer à un achat groupé auprès des fabricants, des centrales d'achats, des grossistes ce qui lui permet ainsi d'obtenir un prix d'achat plus bas en fonction de la quantité négociée comme l'explique ce pharmacien : «C'est-à-dire que le choix final du produit, j'espère qu'ils prennent uniquement parmi les produits actifs, mais après c'est essentiellement quand même les conditions tarifaires qui vont faire qu'ils vont utiliser plutôt celui-là que l'autre... »(entretien 1).

Pour justifier les raisons du choix des produits répulsifs en vente dans les pharmacies différentes raisons ont été énoncées :

- la qualité de leurs produits par rapport à ceux vendus dans d'autres commerces [3] :

«ils sont plus respectueux du derme et de l'épiderme, j'ai jamais vu avec les produits qu'on fait nous des rougeurs alors que dans les commerces quelques fois il y a des soucis, alors c'est pas l'efficacité si vous voulez. du répulsif, mais c'est surtout dans la qualité au point de vue dermatologique, j'ai des clients qui quelquefois avec des produits bas de gammes, ont eu des réactions allergiques, .../(nous) on a des marques qui sont très efficaces dans la durée donc plus économiques aussi » (entretien 8)

- le type de moustiques et l'importance croissante des moustiques dans la région :

«Un laboratoire sérieux, c'est le $5 / 5$, on a Bayer et Cooper, deux laboratoires pharmaceutiques .../ je pense qu'on va finir par avoir des résistances, mais chez nous ça devient important il faut quand même qu'on se protège, avant d'arriver à la moustiquaire... » (entretien 9)

- Le fait également que le produit soit présenté comme ayant été utilisé par les militaires.

La qualité des répulsifs vendus a évolué avec l'implantation des Aedes albopictus. Interrogés sur l'adaptation des produits destinés aux pays «tropicaux» pour les régions tempérées et surtout sur la nocivité éventuelle de ces produits, on peut relever que l'usage et donc la vente de ces répulsifs est justifié par la durée d'action et par l'absence de problèmes liés à l'usage.

«Parce que quand on leur dit que c'est plus actif c'est un truc simple, même ici on vend des trucs tropicaux parce que l'action est meilleure et puis la durée de l'effet est plus longue... Ce sont des produits qui sont élaborés, qui sont surveillés,.../ le produit en officine j'ai confiance en lui, après il y a des produits qui se ressemblent on étudie un peu les produits on les choisit suivant la notoriété commerciale et les conditions (d'achat) du produit» (entretien 7).

Par contre, la nocivité des répulsifs n'est pas envisagée et cette attitude est justifiée par la confiance dans les fabricants : «Celui qui s'occupe des commandes il examine le produit et puis voilà, après c'est le rôle du labo qui commercialise le produit il fait sa promotion .../ On voit un petit peu les formules mais on ne connait pas tout non plus, après on fait confiance aussi à l'industrie qui met ses produits sur le marché, après c'est pas balancé comme ça, moi je fais confiance à l'industriel aussi, le gars il va pas sortir des produits qui vont engendrer des problèmes de santé publique »(entretien 7).

La citronnelle, produit naturel, est même spontanément citée comme étant un produit dangereux selon les usages qui en est fait. Une pharmacienne a signalé la vente des bracelets répulsifs, en expliquant notamment qu'elle en avait utilisé lors de voyages en Afrique du Nord : «Maintenant les bracelets ça marche super bien 》(entretien 9).

Le pharmacien : la dualité commerçant/acteur de santé publique

Le pharmacien se revendique comme acteur de santé publique. Le positionnement est basé sur l'idée que «le pharmacien est ouvert et disponible 7 jours/7 et donne des conseils gratuits sans rendez-vous $\gg$.

Les notions de conseils et d'information sont revendiquées comme étant une fonction importante du métier de pharmacien :

«C'est nous qui vendons les produit, il n'y a pas que nous, mais une grande partie doivent être vendus par les pharmaciens, quand on vend les produits on sensibilise les gens, on leur dit combien de fois faut en mettre on ne va pas dire simplement ben voilà et puis ça fait $8 € 90 »$. (entretien 7)

Il faut toutefois souligner que cette dynamique est actuellement impulsée dans la logique d'une nouvelle convention sur la rémunération des pharmaciens (en cours de discussion à l'assemblée en décembre 2011 et votée fin mars 2012) qui prévoit que les pharmaciens jusqu'à présent rémunérés 《à la boite vendue » verront leur rémunération dépendre pour une partie des conseils qu'ils dispensent (pilule du lendemain, traitement de substitution à la drogue), pour le suivi de certains patients (sous anticoagulants ou asthmatiques), leur participation à des actions de santé publique, et la réalisation d'objectifs tels que la part de génériques délivrés).

Pourtant l'UFC «Que Choisir» a publié une enquête réalisée en France en janvier 2012 dans 14 villes et auprès de 548 pharmacies sur les pratiques des pharmaciens montrant qu'un sur deux ne délivre aucun conseil sur les risques d'interaction médicamenteuse et que le prix d'un même médicament non remboursé passe parfois du simple au quadruple d'une officine à l'autre $[5,6]$.

En matière de santé publique Les pharmaciens mettent également en avant leur habitude de pratiques managériales au sein des pharmacies, ce qui les rend plus aptes à gérer une crise sanitaire par rapport aux médecins qui sont plutôt dans l'exercice d'une pratique individuelle.

Par ailleurs, la faible disponibilité des médecins est évoquée chez les pharmaciens notamment lors de l'épisode de grippe H1N1 et les difficultés de l'exercice individuel en médecine libérale : «les médecins et les infirmières venaient chercher les masques chez nous, il y avait eu à l'époque une réunion chaude à la mairie avec les syndicats pharmaceutiques, l'ordre des médecins, les médecins n'étaient pas capables à l'époque d'aller chercher les masques dans des dépôts en ville.../ on en était arrivé à un système où les médecins et les infirmières pouvaient venir s'approvisionner dans les pharmacies, ça paraissait un truc impossible à réaliser pour les médecins, ils ne pouvaient pas se déplacer, ils ne pouvaient pas aller dans tel endroit, alors 
que nous on est organisés on a des secrétaires on manage des équipes...»(entretien 7).

Parmi les arguments avancés, il y a également celui du pharmacien et de son statut de premier intervenant dans l'itinéraire thérapeutique :

«C'est nous qui avons le premier contact, quand on les envoie chez les médecins et surtout les généralistes, ils ne connaissent rien, donc nous sommes je pense au premier rang parce que nous sommes les premiers que les patients viennent voir quand ils ont été piqués, ils viennent chercher une pommade quelque chose comme ça» (entretien 9). Les pharmaciens considèrent qu'ils ont également une fonction importante d'informations vis-à-vis des voyageurs et expliquent que les recommandations évoluent et que cela les oblige à actualiser leurs connaissances. "C'est nous en premier qu'ils viennent voir quand ils partent à l'étranger pour avoir des conseils et des traitements 》 (entretien 9).

De même, les pharmaciens disent que quand il y a un risque sanitaire signalé les clients se tournent vers les pharmaciens : "Là quand il y a eu l'accident nucléaire, sur le côté comprimés d'iode c'était vers le pharmacien d'officine, ils se sont retournés dessus, là en ce moment c'est les biberons, le coté stérilisation...»(entretien 1)

Néanmoins, ils se disent concurrencés par internet et considèrent qu'ils conservent leur rôle de conseil, mais seulement en deuxième recours :

«D'abord ils vont sur internet, et après ils viennent confirmer ou non, écouter entre guillemets l'avis du professionnel, mais maintenant c'est dans ce sens alors. . . » (entretien 1).

\subsection{L'expérience et la mémorisation des deux cas de dengue autochtones un an auparavant}

Il faut noter que les pharmaciens interrogés n'ont pas été marqués par l'épisode des deux cas de dengue autochtones à Nice. Ils n'en ont pas souvenir, ou une mémorisation assez floue :

«Voilà ils ont démoustiqué, bon on a su qu'il y avait des problèmes, mais moi je ne savais pas qu'y avait eu des cas de dengue autochtones $\gg$ (entretien 9).

Un autre dira: «c'est passé complètement inaperçu. Au comptoir, moi j’ai eu aucun écho » (entretien 8).

Mais, il précisera que le faible impact des deux cas de dengue dans la population niçoise est lié à la moindre attention accordé par la presse à ces deux cas, par rapport aux informations médiatiques durant l'épisode de chikungunya à la Réunion : «Oui on a demandé des documents, et ça dépend aussi s'il y a un impact, la dengue effectivement, mais bon les gens pensent que la dengue c'est tropical, par contre le chikungunya qu'on peut pas attraper ici qu'on attrape ailleurs et qu'on peut développer effectivement ici alors là ça ils sont tous «je vais choper le chikungunya, je vais choper le chikungunya », non, pour le moment il y a aucune preuve qu'on puisse attraper le chikungunya avec des moustiques autochtones, ça dépend comment la presse monte l'affaire !... » (entretien 8).

Il rajoutera : «Y a pas eu grand-chose, ça n'a pas été monté en épingle comme le chikungunya de 3,4 ans .../ reprenez la presse des années 2006-2007 sur le chikungunya, c'est l'Apocalypse, ils vous foutent une trouille pas possible, c'est tout, les gens ils suivent ce que les médias transmettent. Si vous regardez les articles de l'époque, les gens ils nous interrogeaient tous les jours, ils avaient la trouille, mais parce qu'on leur avait foutu la trouille» (entretien 8).

\section{Formation, informations : quelles adéquations et adaptations à envisager}

Connaissances des maladies tropicales, expérience de conseils formulés

Au-delà du faible impact, du moins en termes de mémorisation de l'épisode de dengue et même de chikungunya dans le Var, les pharmaciens rencontrés ne se disent pas confrontés aux maladies tropicales. Une pharmacienne se dit pas suffisamment formée et peu intéressée expliquant que ce sont des questions éloignées de ces questionnements professionnels quotidiens : «et les médecins ne sont pas du tout informés, et nous ne sommes jamais suffisamment informés .../On a des formations continues bon pas spécialement sur la dengue ou sur le chikungunya parce que ce n'est pas quelque chose qui nous passionne.../ ce ne sont pas des problèmes de chez nous ça jusqu'à présent » (entretien 9).

Un autre précise : « Je suis sorti en 1977 alors vous vous rendez compte maintenant elle est facile la réactualisation des connaissances, bon je ne serai pas un professeur de parasitologie, mais si je vais sur internet j'aurais des informations. Pour moi c'est une source d'infos j'y vais facilement, après je peux faire une démarche j'irais dans une bibliothèque universitaire de Nice. Je ne suis pas très compétent (sur les arboviroses), mais après je m'informerais et je saurais diriger le client »(entretien 7).

Dans plusieurs cas, les entretiens étaient menés avec des professionnels qui au cours de l'entretien ont fait état de nombreuses dynamiques professionnelles, syndicales et humanitaires. On peut en conclure qu'au regard de la faible mémorisation de l'épisode de dengue un an auparavant comme de l'état des connaissances dont ils font mention, il serait intéressant dans le cas d'une étude plus approfondie et surtout quantitative de faire un point plus précis sur les connaissances des pharmaciens en matière de maladies tropicales, car les savoirs restent assez généraux chez des professionnels censés informer et conseiller la population.

«Vous connaissez les symptômes : Non, à part la forte fièvre c'est tout» (entretien 9).

\section{La réception et la diffusion des informations dans les pharmacies}

Les documents d'information (flyers) sont déposés et à disposition dans les officines.

Certains disent : «Ça reste sur le comptoir et ça part à la poubelle, parce que les gens ils ne les prennent pas alors ils regardent, on en a eu, enfin peut-être pas ceux-là pas ceux du Conseil Général, mais des labos le même genre de choses, les gens se sentent peu concernés»(entretien 8). 
En général, les pharmacies reçoivent des affiches à mettre en vitrine, et une invitation à commander des prospectus quand ils ne leur sont pas livrés en même temps.

«Ils nous envoient des affiches, ils nous envoient des prospectus comme ça, on avait une affiche qu'on avait mis devant

- Et les gens ça provoque des questions?

- Très peu.

- Ils prennent les prospectus?

- Très peu, ils prennent chez nous des livres comme Bien-Etre etc., mais par contre les prospectus ils n'en prennent pas trop » (entretien 9).

En matière d'informations professionnelles les pharmaciens sont abonnés à différentes revues professionnelles : «Le Quotidien», «le Quotidien du pharmacien», «Pharmaciens de France», «les Nouvelles pharmaceutiques », la revue «Prescrire» surtout orientée sur les médicaments... Différents sites web destinés aux professionnels, existent également. Par ailleurs, les pharmaciens reçoivent directement sur leur site toutes les alertes du ministère de la santé. On constate qu'internet est surtout présent dans le bureau du pharmacien principal, et qu'ils ne l'ont pas tous au niveau du comptoir.

Ils font le constat néanmoins qu'ils reçoivent quotidiennement beaucoup d'informations et qu'ils doivent faire un tri : "puis il y a tellement d'informations, tellement de sollicitations, vous savez il y a un tri qui doit se faire, il y a tellement d'informations qui arrivent quotidiennement $\gg$.

\section{Privilégier la rapidité en matière d'informations et d'alertes sanitaires}

Il faut souligner le décalage temporel entre l'information des pharmaciens et celui de la presse. Le discours sur la maladie est produit par les médias et les autorités sanitaires. Pour ce qui concerne les maladies vectorielles il semble avoir été prégnant lors de l'épidémie de chikungunya à la Réunion, et lors de l'installation des premiers moustiques tigres, venus d'Italie, dans la région.

Mais, on peut souligner le discours récurrent des professionnels de santé sur le retard entre l'information diffusée par les médias et l'information professionnelle des pharmaciens (et aussi des médecins) qui disent souvent l'avoir appris d'abord par les médias.

«Et quand ils ne savent pas trop ils viennent chez. le pharmacien, et le problème c'est que le pharmacien la plupart du temps il l'apprend quasiment en même temps. Le bisphénol, tout le monde a découvert ça aux nouvelles, le soir, donc les pharmaciens qui avaient vu le JT la veille, ils étaient plus ou moins au courant mais ils n'avaient pas plus d'infos que le patient lambda» (entretien 1).

Il faut noter de récents changements mentionnés par plusieurs : «Jusqu'à l'affaire Servier, dans ce type d'alertes, nous on était avertis souvent par la presse, avant d'être avertis par le canal classique, et heureusement l'AFFSAPS a disparu, c'est maintenant la Haute Autorité de Santé qui a changé la donne, ce qui fait que maintenant quand une alerte est lancée on la reçoit sur nos écrans informatiques en même temps qu'elle est donnée pour tous les intervenants de santé, mais ça date d'il y a trois mois ça, l'an dernier encore on était avertis souvent par la presse ou par nos grossistes, par un flyer qui arrivait 24, 48 ou 72 heures après... ! » (entretien 8).

Les pharmaciens se disent inscrits par les listes professionnelles et syndicales sur la liste de diffusion «DGS urgent» qui envoie des informations en cas d'alertes, épidémiques ou de problèmes de santé :

«Maintenant oui, oui ça on le reçoit sur internet sur nos écrans, on vient justement de recevoir un truc demandant nos numéros de portables personnels, ça c'est pour l'iode» (entretien 8).

Le rôle d'information des vitrines est mis en évidence par les pharmaciens : «Oui, que l'information puisse passer par nous parce que c'est facile, il y a des vitrines on peut exposer, on peut faire passer des messages s'il y a un caractère de gravité, parce que nos vitrines les gens les regardent. Si on les change c'est qu'elles ont un impact quand même, nous on s'attache à faire des vitrines elles doivent donc attirer le chaland et par ce truc on peut faire passer des messages $\gg$ (entretien 7).

Malgré l'installation d'Aedes albopictus dans la région PACA et deux cas de dengue et de chikungunya autochtones, le risque d'épidémie ne semble pas constituer pour les pharmaciens de la région niçoise un sujet de préoccupation. Le même constat peut d'ailleurs être fait pour les médecins généralistes qui ont été rencontrés lors de la même enquête.

Ce travail fait toutefois apparaitre le rôle d'acteur de santé publique que pourraient être amenés à jouer les pharmaciens. Du fait de leur proximité avec la population, et sous réserve de la mise en place de réseaux d'information et de formation efficaces, ils sont en effet en situation d'être des relais pour participer à des campagnes de prévention. Il nous semble donc que ce travail à portée exploratoire devrait être poursuivi à l'échelle de la métropole sur un échantillon représentatif de professionnels de santé, d'autant plus, que Aedes albopictus est en train de s'implanter sur tout le territoire.

Cette étude a été réalisée dans le cadre du projet FAVELA (Facteurs sociaux de vulnérabilité face à une épidémie liée à un arbovirus) et financée par 1'IMMI en 2011.

Remerciements : Nous remercions les professionnels de santé qui ont accepté de s'entretenir avec nous sur cette question et tout particulièrement le Docteur Pascal Delaunay - entomologiste médical - parasitologue du CHU Nice/Hopital 1'Archet pour son aide et ses conseils.

\section{Références}

1. ARS PACA, 2012, Schéma régional de prévention. Volet relatif à la veille, l'alerte et la gestion des urgences sanitaires, 90 p, consulté le 26 avril 2012 http://www.ars.paca.sante.fr/fileadmin/PACA/ Site_Ars_Paca/Projet_regional_de_sante/Docs_officiels_ fevrier2012/2012-01-

2. MEDQUAL, Etat des lieux dengue et chikungunya en France, Octobre 2010, pp.1-4, (2010). 
3. A. Marchet, Les arboviroses tropicales émergentes en France (Dengue et chikungunya. Etude du rôle du pharmacien d'officine dans la lutte antivectorielle, Thèse de doctorat en pharmacie, Université Angers, 192 p. (2011).

4. D. Philipps, Extension des moustiques vecteurs : le cas
d'Aèdes albopictus, Thèse de doctorat en pharmacie, Université de Strasbourg, 79 p. (2009).

5. N. Ducatez, Douloureux constat, Que Choisir, Avril, n 502, p.30. (2012).

6. [QUE CHOISIR, Pharmacies. Prix et conseils, le grand bazar!, Avril, n502, 28-30. (2012). 